\title{
Investigation of Psychometric Properties of Rumination-Reflection Scale
}

\section{Davood Manavipour*, Arezo Shahhosieni}

Department of Psychology, Garmsar Branch, Islamic Azad University, Garmsar, Iran

\section{A BSTRACT}

Introduction: Rumination is defined as a series of repetitive thoughts to the negative mood or events and reflection is dispositional self-attentiveness motivated by intellectual interests. The purpose of the present study was to develop a Persian version of RuminationReflection Questionnaire designed by Trapnell \& Campbell and examine its reliability and validity. Materials and Methods: A group of 143 students from Azad University, Garmsar Branch were selected by available sampling during 2014-2015. Results: The findings showed that reliability coefficients for the 11 remaining items of RuminationReflection scale were 0.73 , which determine $71.78 \%$ of the variance of rumination test with 3 factors of reflection, introspection, and brooding. Conclusion: The Persian version of questionnaire can be used to measure the rumination among Iranian subjects.

*Corresponding Author: Davood Manavipour

E-mail: Manavipor53@yahoo.com 


\section{بررسى شاخصهاى روانسنجى مقياس نشخوار فكرى -تأمل}

\section{داود معنوى ثبور”، آرزو شاه حسينى}

كروه روانشناسى، واحد كرمسار، دانشكاه آزاد اسلامى، كرمسار، ايران

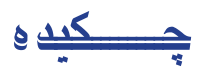

مقدمه: نشخوار فكرى بهعنوان يكسرى از افكار تكرارى نسبت به خلق منفى يا رويدادهادها تعريف شده است

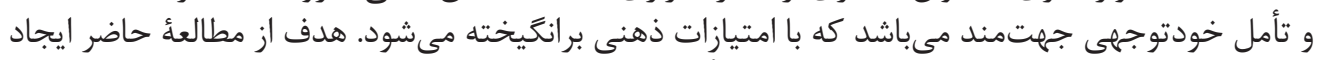

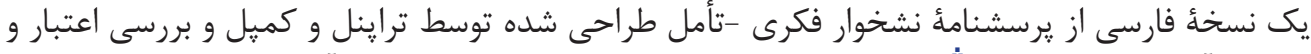

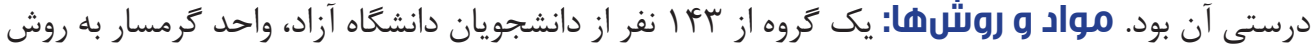

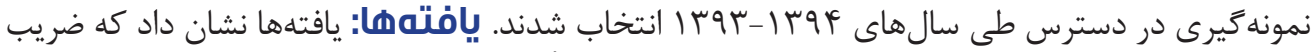

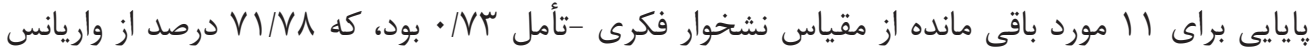

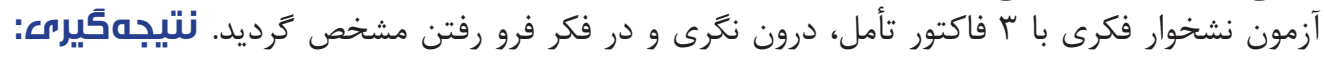

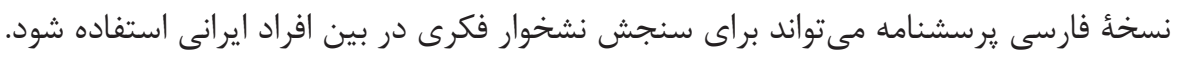

ا

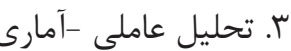

" نويسنده مسئول: داود معنوى يور : آدرس الكترونيكى: Manavipor53@yahoo.com 


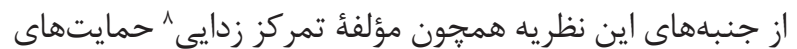

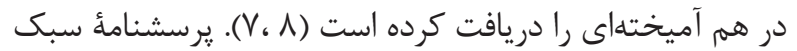

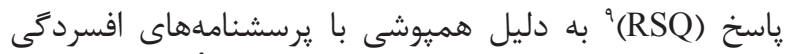

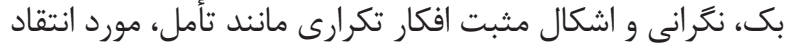

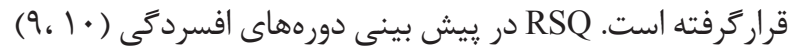

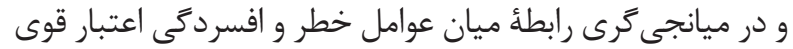

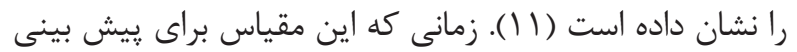

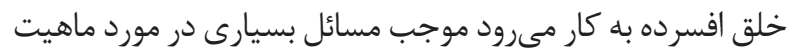

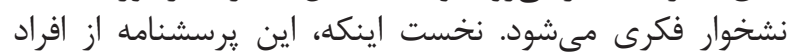

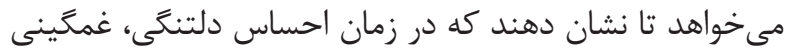

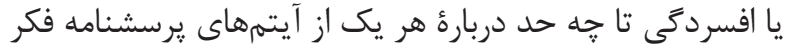

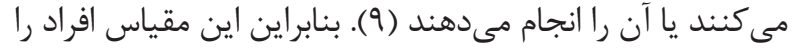

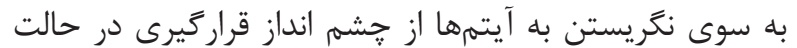

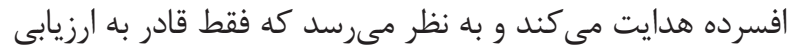

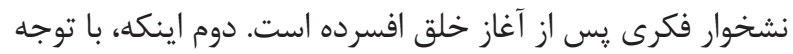

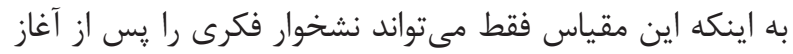

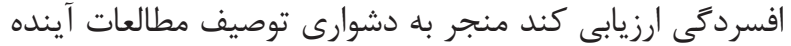

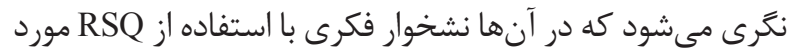

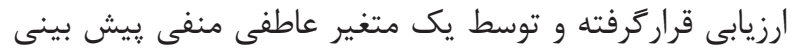

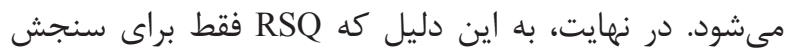

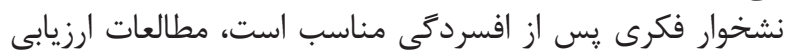

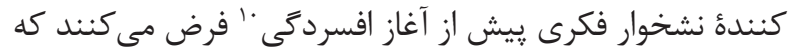

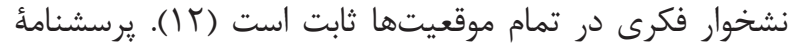

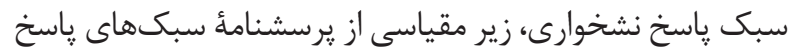

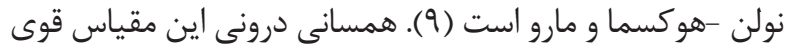

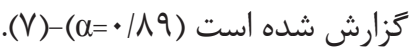

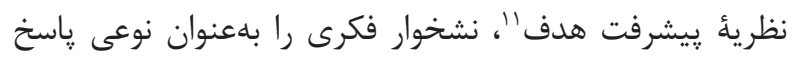

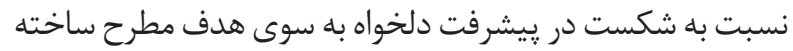

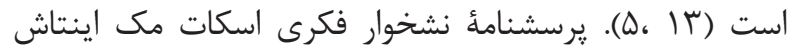
(SMRI)

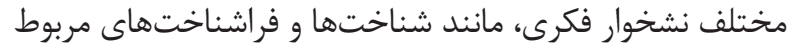

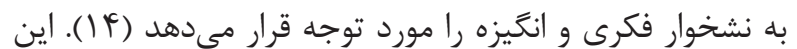

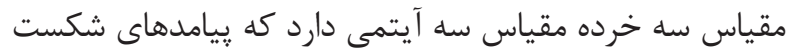

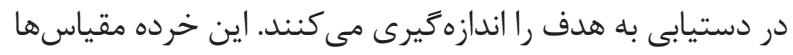

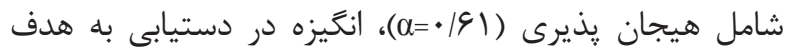

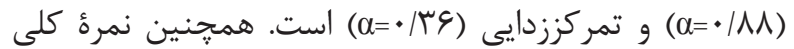

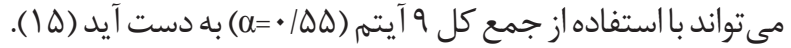
نظرئ عملكرد اجرايى خود نظم بخش (S-REF)" ديدكاه

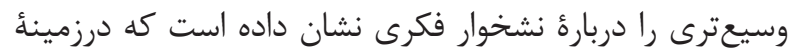

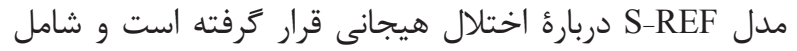

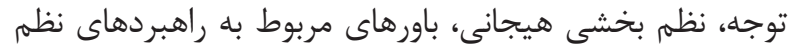
بخشى هيجانى و تعامل ميان سطوح مختلف فرايندهائ بار هرئ شناختى

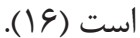

\footnotetext{
${ }^{1}$ Rumination

${ }^{2}$ Martin

${ }^{3}$ Tesser

${ }^{4}$ Siegle

${ }^{5}$ Depression

${ }^{6}$ Response styles theory

${ }^{7}$ Nolen and Hoeksema
}

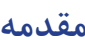

در سالهاى اخير بررسى الكوهاى تفكر ناساز كارانه در اختلالات آنات

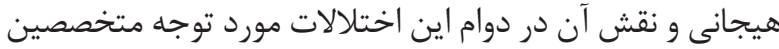

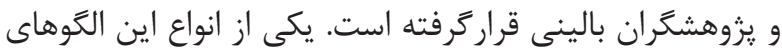

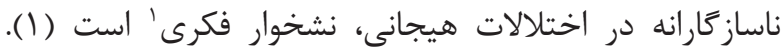

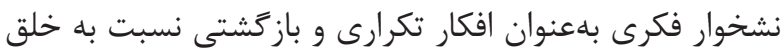

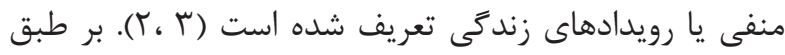

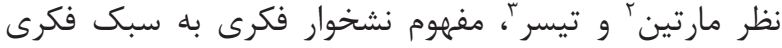

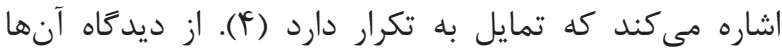

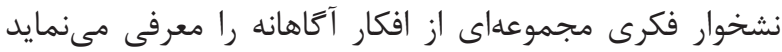

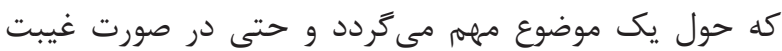

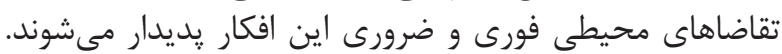

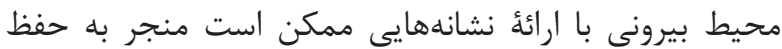

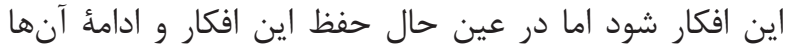

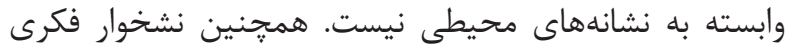

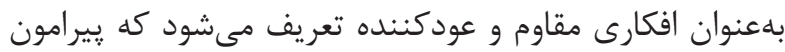

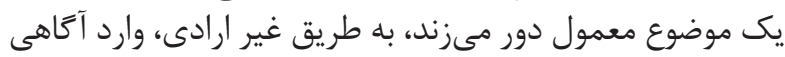

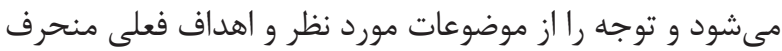

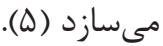

علاوه بر تعاريف مختلف نشخوار فكرى كه به آنها اشاره شد،

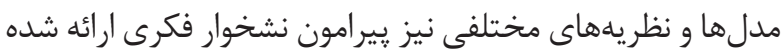

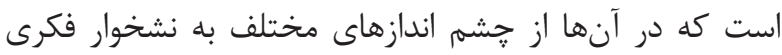

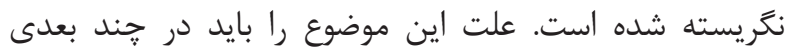

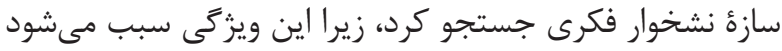

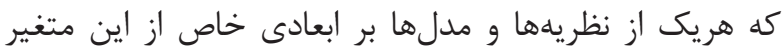

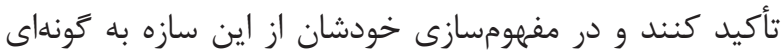

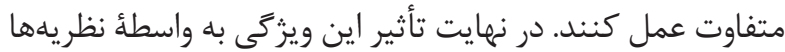

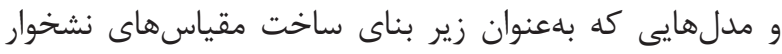

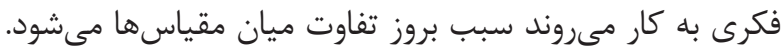

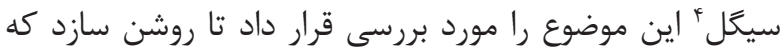

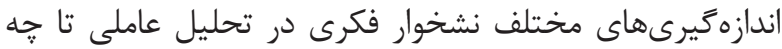

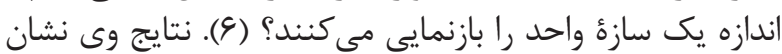

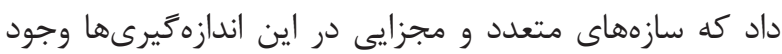

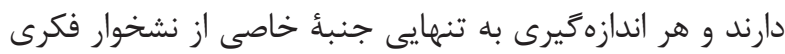

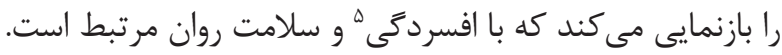

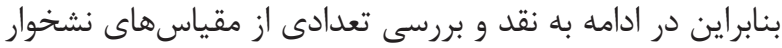

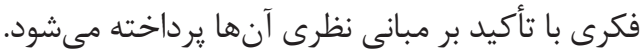

نيرومندترين نظريئ نشخوار فكرى، نظريئ سبك ياسخ (RST)

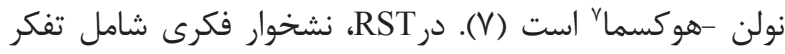

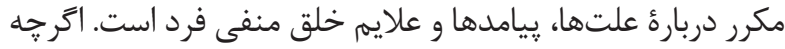

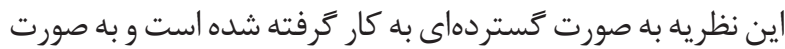

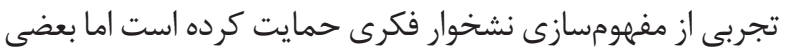

\footnotetext{
${ }^{8}$ Distraction

${ }^{9}$ Response styles questionnaire

${ }^{10}$ Pre-depression

${ }^{11}$ Goal progress theory

${ }^{12}$ Scott mcIntosh rumination inventory

${ }^{13}$ Self-regulatory executive function
} 


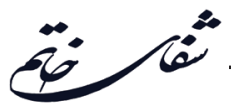

طرحهاى آزمايشى كه تفسير منفى و يا ناميدى را يكيارجه نمى سازند با نابسند

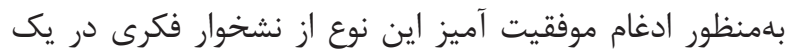

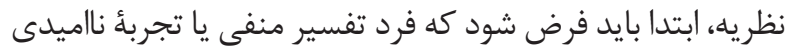

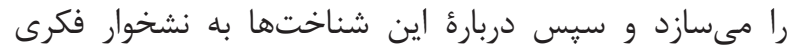

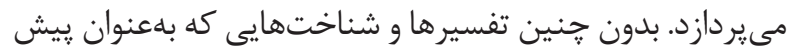

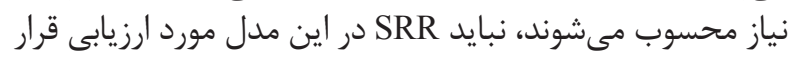

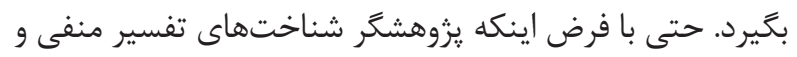

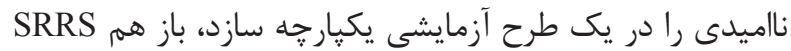

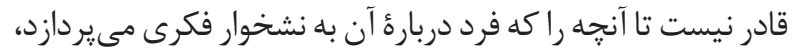

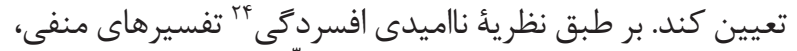

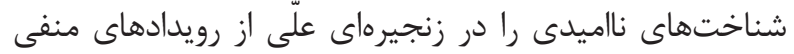

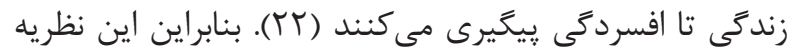

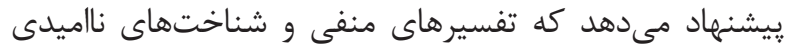

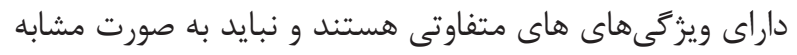

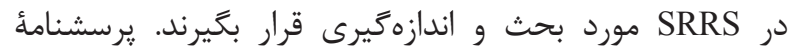

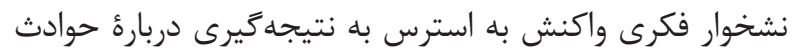

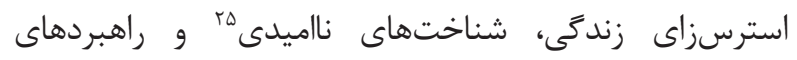

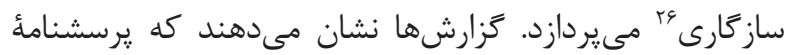
SRRS

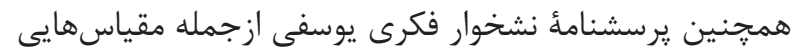

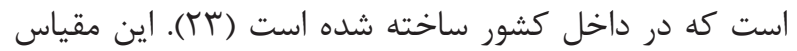

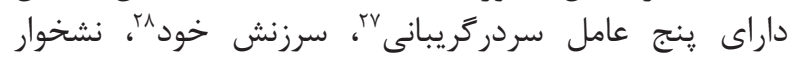

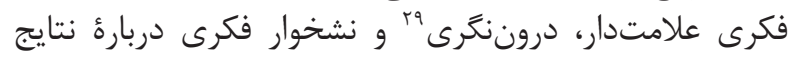

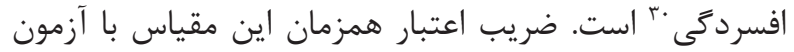

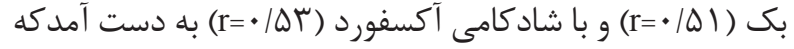

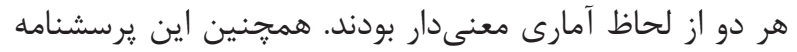
از ضريب آلفاى كرونباخ ( 1 ( •) برخوردار است.

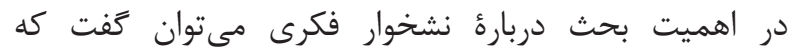

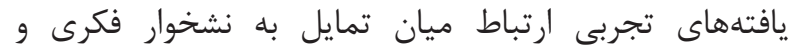

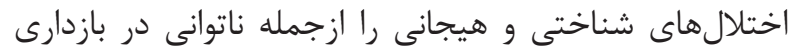

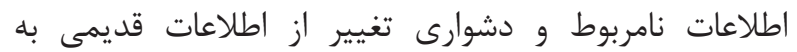

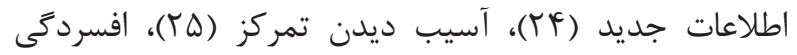

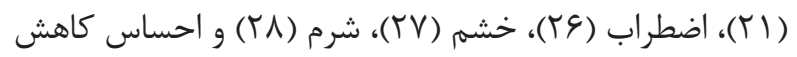

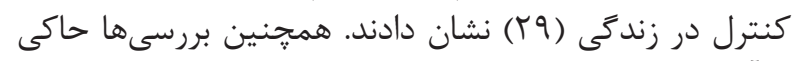

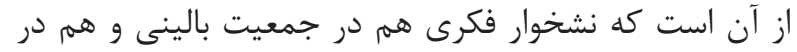

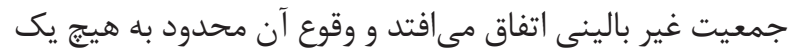

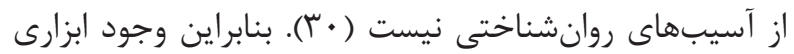

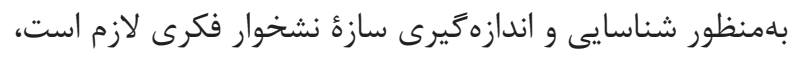

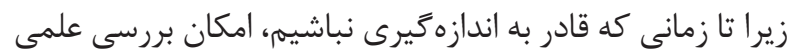

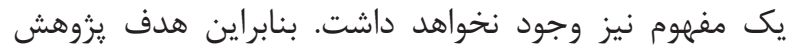

\footnotetext{
${ }^{14}$ Wells

${ }^{15}$ Matthews

${ }^{16}$ Positive belief rumination scale

${ }^{17}$ Meta cognition questionnaire

${ }^{18}$ Negative belief rumination scale

${ }^{19}$ Conway

${ }^{20}$ Rumination on sadness

${ }^{21}$ Alloy

${ }^{22}$ Stress-reactive rumination
}

ولز" و و متيوس ها در مدل S-REF نشان دادند كه عقايد افراد

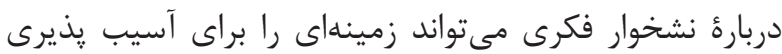

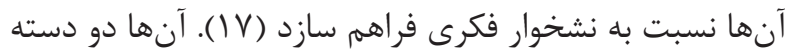

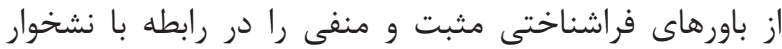

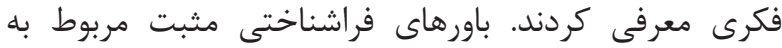

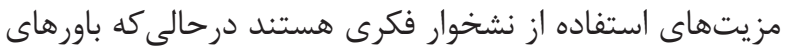

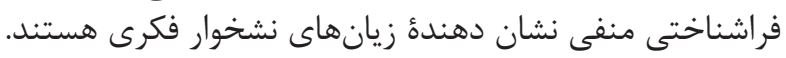

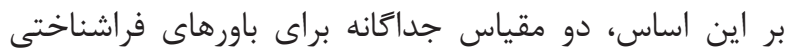
مثبت و منفى ساخته شده است (1) (1).

مقياس باورهاى مثبت نشخوار فكرى (PBRS)" به اندازمخيرى

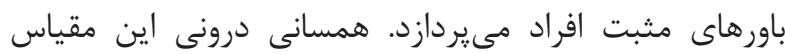

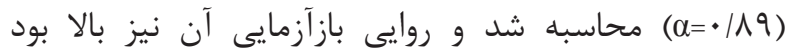
فراشنا

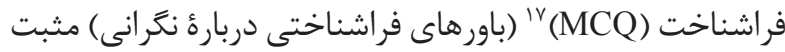

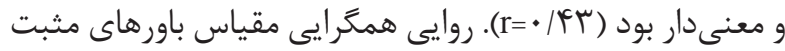

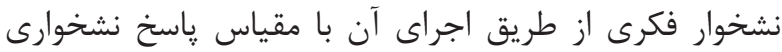

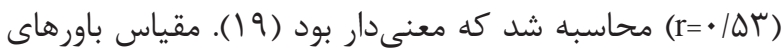

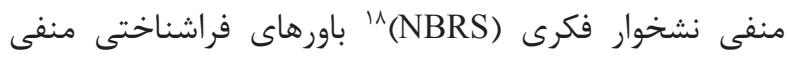

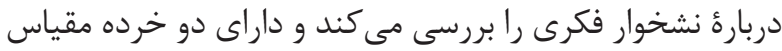

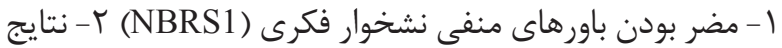

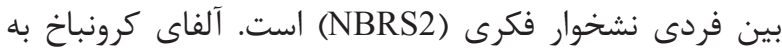

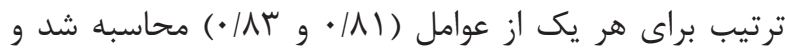

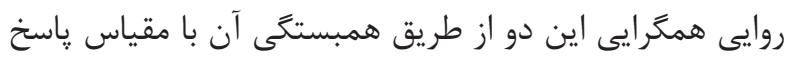

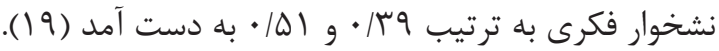

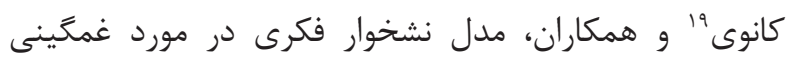
(RSS)

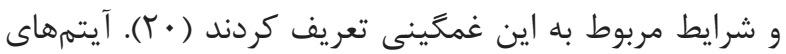

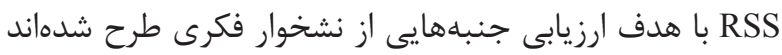

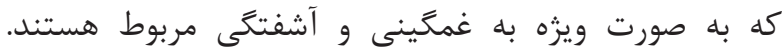

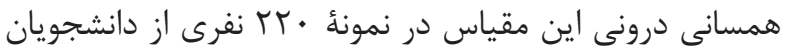

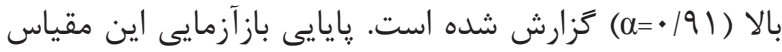

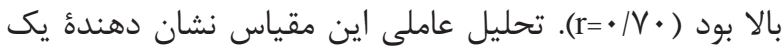

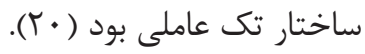

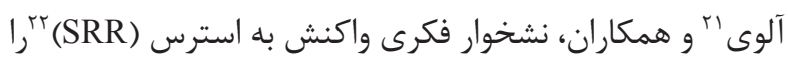

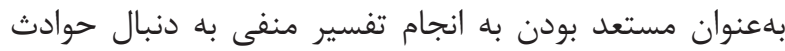

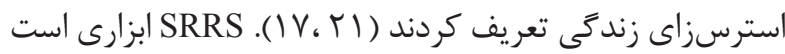

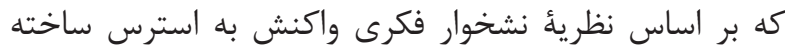

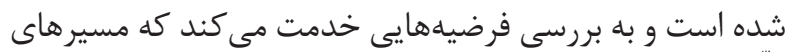

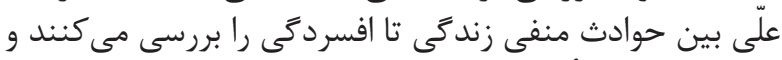

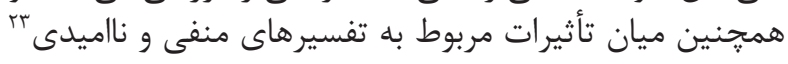

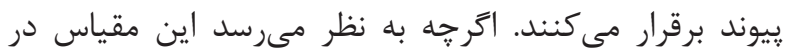

\footnotetext{
${ }^{23}$ Hopelessness

${ }^{24}$ Hopelessness theory of depression

${ }^{25}$ Hopeless cognitions

${ }^{26}$ Coping strategies

${ }^{27}$ Brooding

${ }^{28}$ Self-blame

${ }^{29}$ Introspection

${ }^{30}$ Rumination about depression results
} 


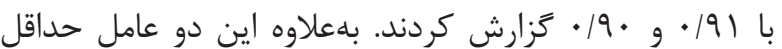

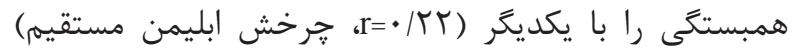

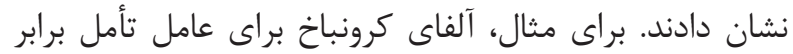

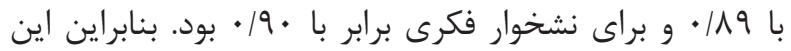

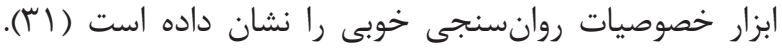

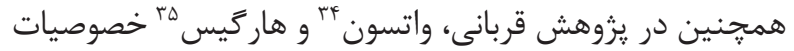

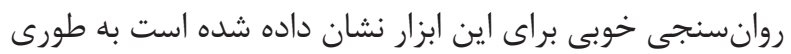

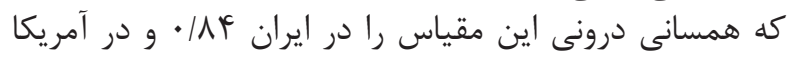

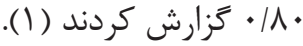

$$
\text { روش اجرا و تحليل }
$$

يرسشنامههاى يزوهش به شيوه خودگزارش دهى تكميل شدند.

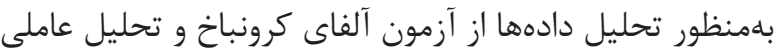

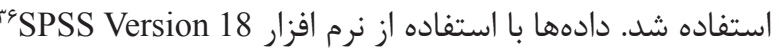
تجزيه و تحليل شد.

بافته ها

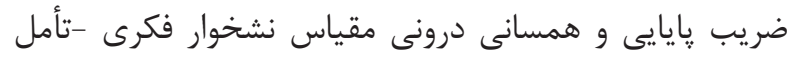

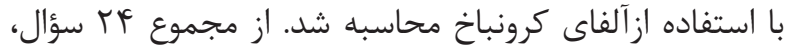

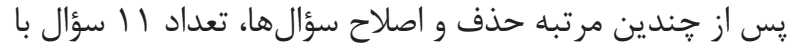

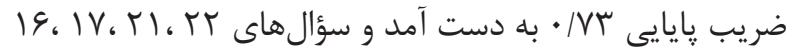

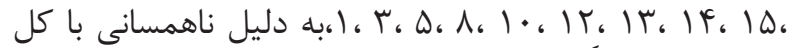

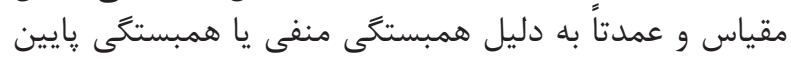
حذف شدند (جدول ( ).

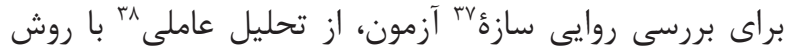

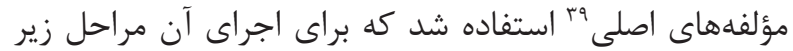
طى شد: مؤه

براى بررسى مناسب بودن دادهها بهمنظور تحليل عاملى، ابتدا

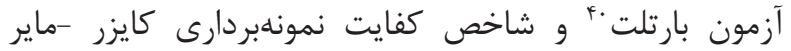

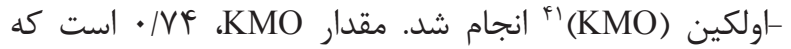

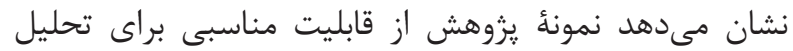

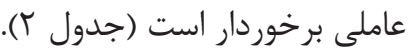
در مرحلة بعد از تحليل عاملى سهمم هر يك از عامل ها يا ارزشهاى

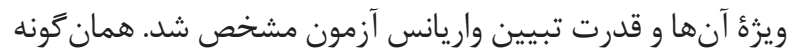

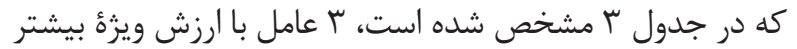

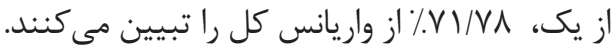
براى تعيين همبستخى هر سؤال با هر عامل، ماتريس همبستخى

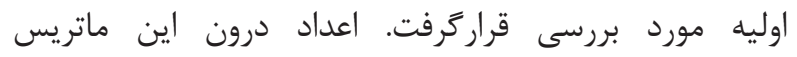

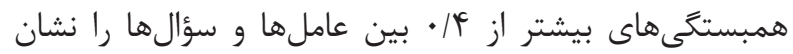

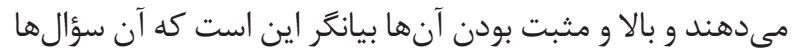

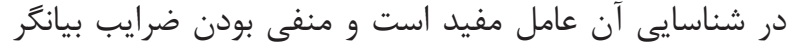

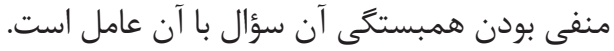

\footnotetext{
${ }^{31}$ Rumination-reflection questionnaire

${ }^{32}$ Trapnell and Campbell

${ }^{33}$ Rumination-reflection scale

${ }^{34}$ Watson

${ }^{35}$ Hargis

${ }^{36}$ Statistical package for social science
}

حاضر بررسى شاخصهاى روانسنجى يرسشنامئ نشخوار فكرى

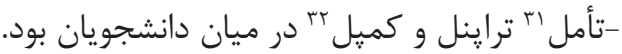

مواد و روشها جامعه، نمونه و روش نمونه

جامعهُ آمارى يروهش حاضر متشكل از كلئُ دانشجويان دانشعاه

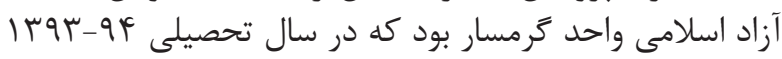

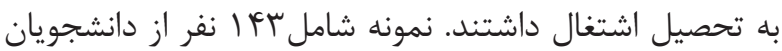

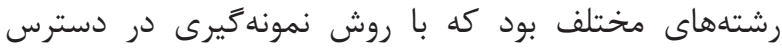

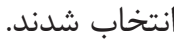

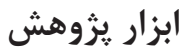

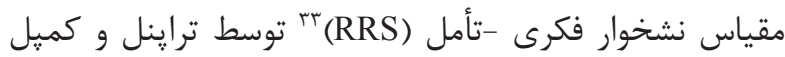

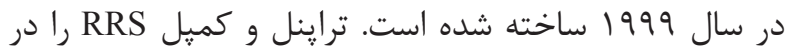

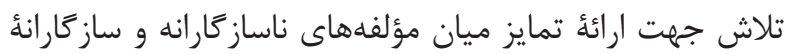

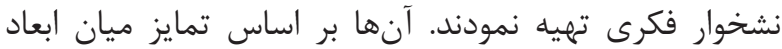

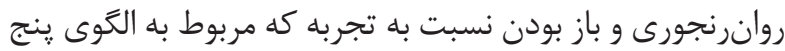

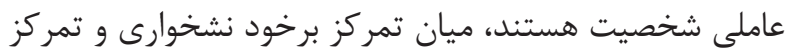

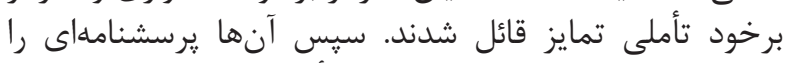

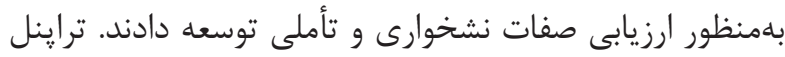

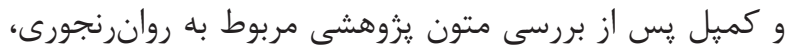

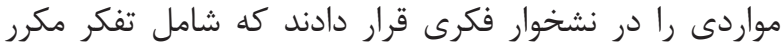

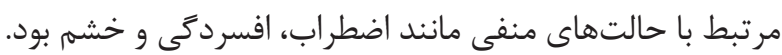

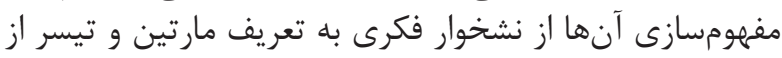

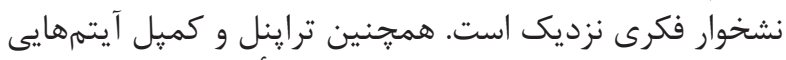

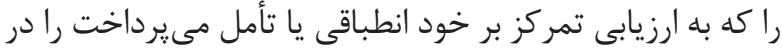

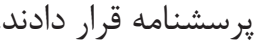

آيتمهاى خرده مقياس تأمل، صفات مربوط به باز بودن نسبت

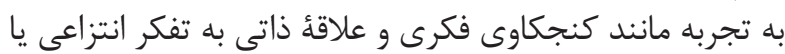

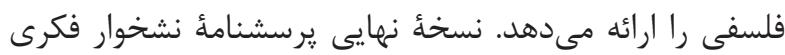

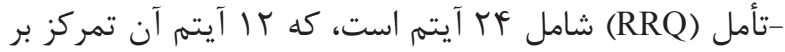

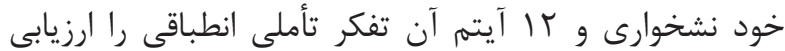

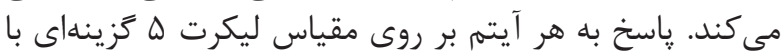

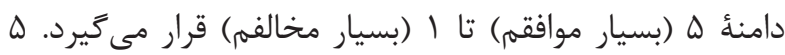

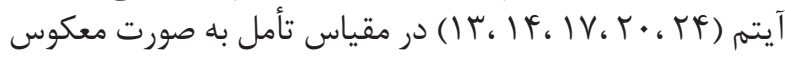

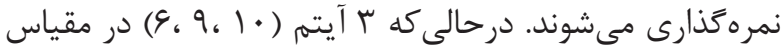

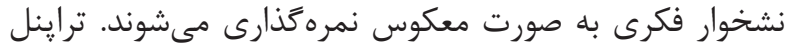

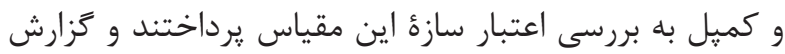

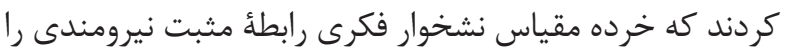

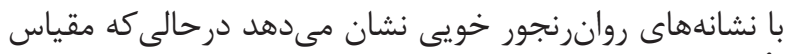

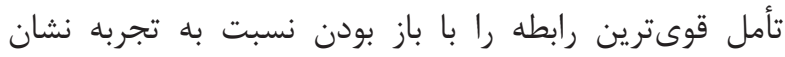

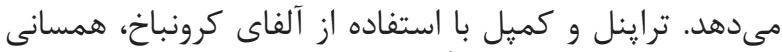

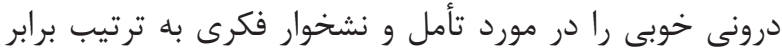

\footnotetext{
${ }^{37}$ Construct validity

${ }^{38}$ Factor analysis

${ }^{39}$ Principle components method

${ }^{40}$ Bartlett's test

${ }^{41}$ Kaiser-Meyer-Olkin
} 


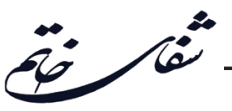

جدول ا- ضريب پايايى مقياس نشخوار فكرى -تأمل.

\begin{tabular}{|c|c|c|c|c|}
\hline ميانكَين در صورت & واريانس در صورت & همبستخئ هر كريه با كل & حذف كَّريه & شمارة: تويها \\
\hline \&q/ $\wedge \vee f I$ & ᄉ१४१F/GVF & •/ $/ \Delta \Delta r$ & $\cdot|9 \wedge|$ & Qr \\
\hline$q 9 / r \cdot r \wedge$ & 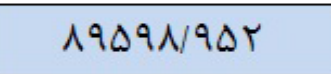 & $\cdot / \Delta \Delta r$ & $\cdot|8 \wedge|$ & Qf \\
\hline GQ/rqसG & 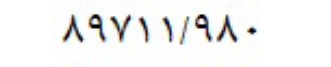 & $\cdot|\Delta \Delta|$ & . /8Nt & Qq \\
\hline GN/qFFI & 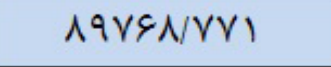 & $\cdot / \Delta \Delta \cdot$ & . /8Nt & QV \\
\hline VD/ATQY & $\mid r F \cdot q V / \cdot r V$ & $\cdot 1 \cdot 1 \cdot$ & ./Vrq & Q9 \\
\hline$v \varepsilon / \Delta \cdot r \Delta$ & ITF.FY/AKQ & $\cdot / \cdot \mathrm{V} \cdot$ & ./Vrq & Q11 \\
\hline धQ/TrAV & 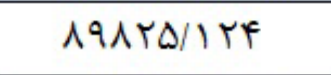 & $\cdot / \Delta F \wedge$ & . /8NT & Q\^ \\
\hline VG/VGYT & | r & (11 & ./Vru & Q19 \\
\hline GQ/TrIV & $\Lambda ৭ \vee F T / T \cdot F$ & $\cdot / \Delta \Delta \cdot$ & . /8Nt & Qr. \\
\hline Vद/f। १९ & $|r f| r / \wedge \& \Delta$ & $-\cdot /$ Tf & ./Vrq & QRT \\
\hline VE/TFYV & Irf. $\Delta F / g q r$ & $\cdot / \Delta \Delta$ & •/Vrq & QYF \\
\hline 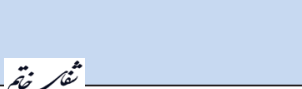 & تعداد يرسش ها= & & س س/آلفا & تعداد افراد=سF \\
\hline
\end{tabular}

جدول r- آزمون كايزر -ماير -اولكين و آزمون بارتلت.

\begin{tabular}{|c|c|}
\hline - NFFF & آزمون كفايت نمونهبردارى كايزر -ماير -ـولكين \\
\hline rVG/94人 & آزمون كرويت بارتلت -تقريب كا -اسكوثر \\
\hline$\Delta \omega$ & درجهُ آزادى \\
\hline$\cdot 1 \cdots 1$ & سطح معنى دارى \\
\hline
\end{tabular}

جدول r- قدرت تبيين واريانس آزمون نشخوار فكرى -تأمل.

\begin{tabular}{|c|c|c|c|c|c|c|c|c|c|}
\hline \multicolumn{3}{|c|}{ ارزشهاى استخراج شده يس از خرخش } & \multicolumn{3}{|c|}{ ارزشهاى ويثرةُ بزرَّتر از 1} & \multicolumn{3}{|c|}{ ارزشهاى ويرةً اوليه } & \multirow[b]{2}{*}{ عاملها } \\
\hline كل & مقدار درصد & تراكمى درصد & كل & مقدار درصد & 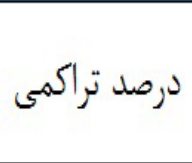 & كل & مقدار درصد & درصد تراكمى & \\
\hline$r / \cdot \psi_{l}$ & TV/GFY & TV/GFY & $r / 1 \cdot 0$ & KN/TYK & KN/TKM & $r / l \cdot \Delta$ & TN/TKM & KN/YTH & 1 \\
\hline$r / r^{\mu}$ & rV/FAY & $\Delta \Delta / / Y F$ & $\mathrm{r} / \cdot 11$ & TV/T\&q & $\Delta \Delta / \Delta q Y$ & $4 / \cdot 11$ & $r V / T G q$ & $\Delta \Delta / \Delta q T$ & $r$ \\
\hline I/Arr & $18 / 909$ & VI/VAr & $1 / V \wedge 1$ & $18 / 191$ & VI/VAY & I/VAI & $18 / 191$ & VI/VAY & $r$ \\
\hline
\end{tabular}




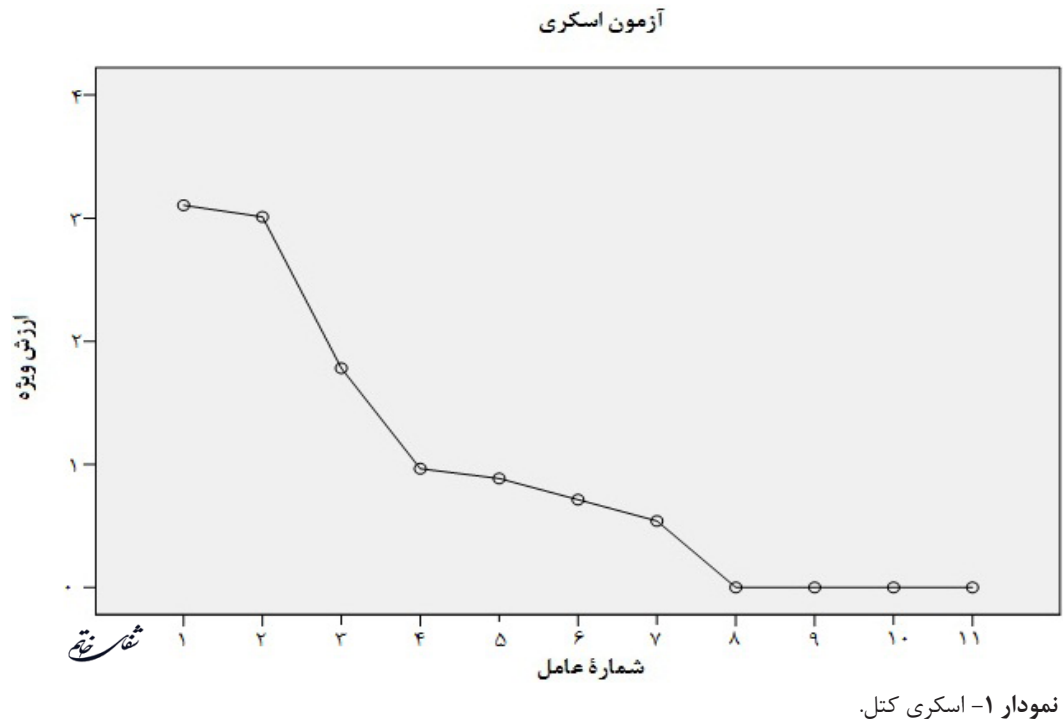

اصلاح شدهاند و در بهترين حالت خود قرار كرفتهاند (جدول \&).

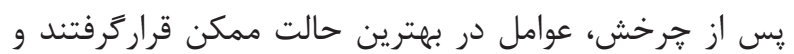

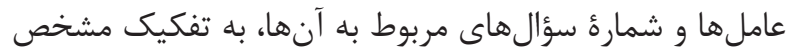

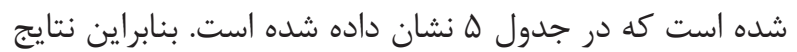

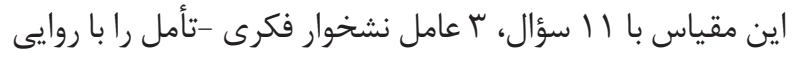

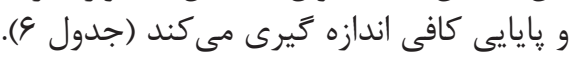

دادهاى ييش از جرخش در ابتدايىترين حالت خود قرار دارند و

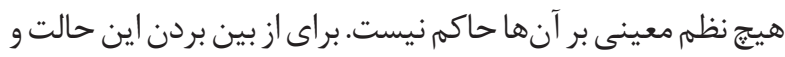

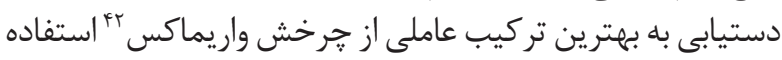

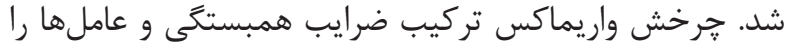

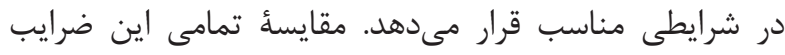

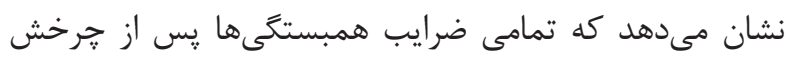

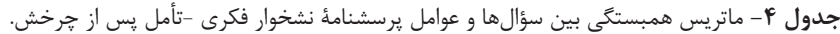

\begin{tabular}{|c|c|c|c|}
\hline \multicolumn{4}{|c|}{ عامل هاى يرسشنـامه } \\
\hline$r$ & $r$ & 1 & \\
\hline & & ./११४ & Qf \\
\hline & & ./११४ & Qr \\
\hline & &.$/ 998$ & Q\& \\
\hline & ./१९१ & & Qr. \\
\hline & ./१११ & & QV \\
\hline & ./१११ & & Q\^ \\
\hline$\cdot / \vee \wedge 1$ & & & $Q^{9}$ \\
\hline.$/ V Y r$ & & & Qrr \\
\hline.$/ 94 \lambda$ & & & Q11 \\
\hline.$/ r \Delta V$ & & & Q19 \\
\hline $\begin{array}{l}-\cdot / r F V \\
\\
\end{array}$ & & & QrF \\
\hline
\end{tabular}

جدول هـ شماره سؤال هاى يرسشنامة نشخوار فكرى -تأمل با هر عامل.

\begin{tabular}{|c|c|c|c|}
\hline شمارهُ سؤال ها & نام عامل & شماره عاملها & رديف \\
\hline r. r. G & ت تأمل & اول & 1 \\
\hline V. IA. Y. & درون نكرى & دوم & r \\
\hline 11. 19. T., Tr & در فكر فرو رفتن & سوم & r \\
\hline
\end{tabular}

${ }^{42}$ Varimax rotation 
جدول 9- آزمون نشخوار فكرى -تأمل يِ از بررسى اعتبار و روايى.

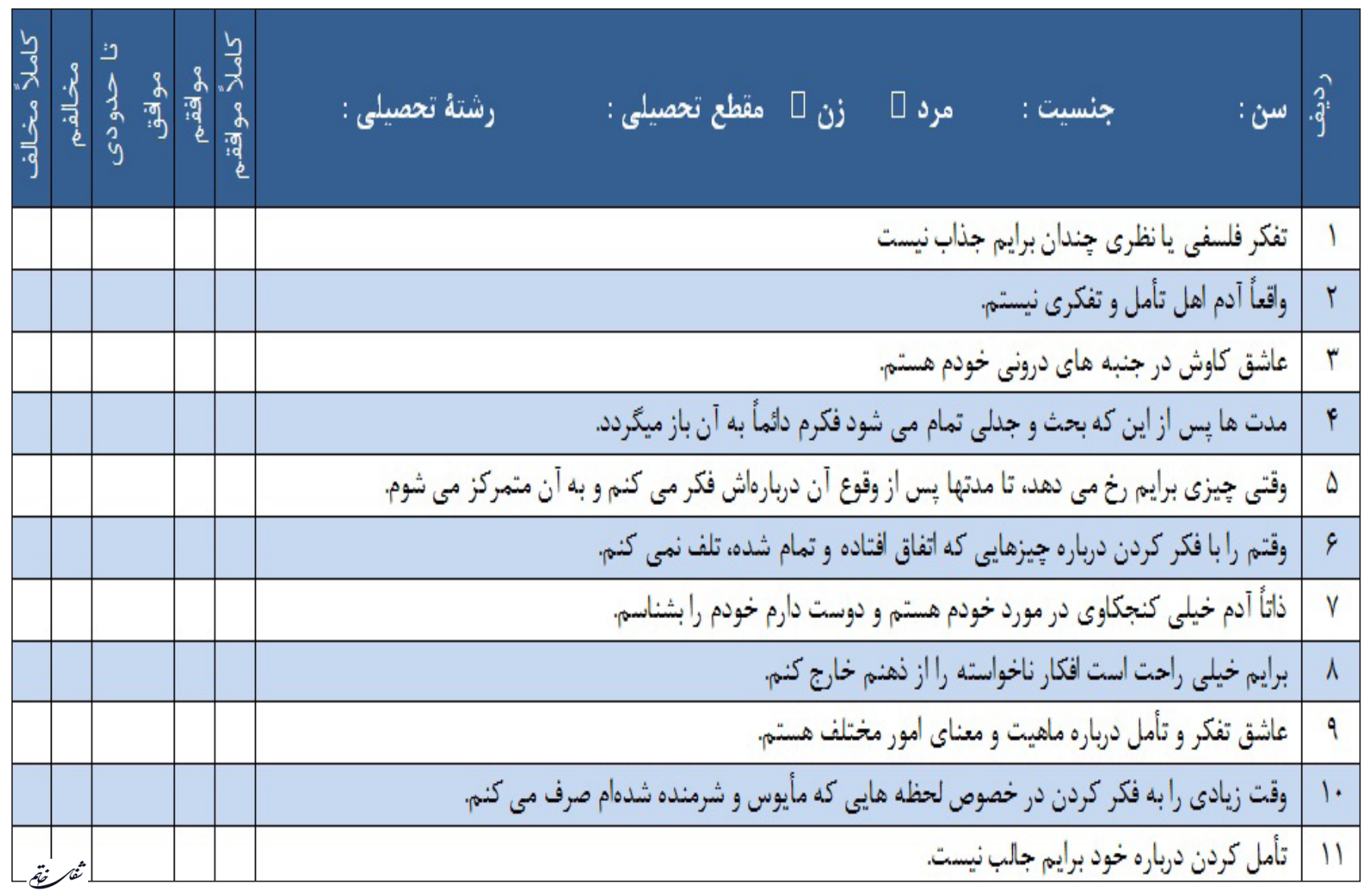

مقياس نشخوار فكرى -تأمل از روايى مناسبى برخوردار است.

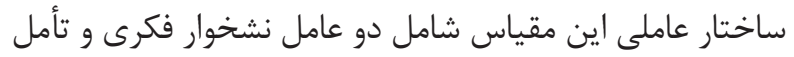

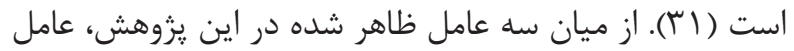

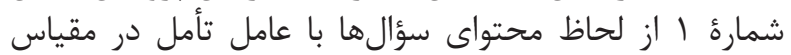

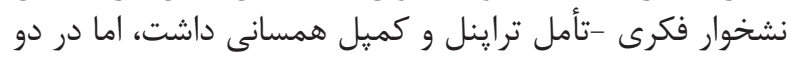

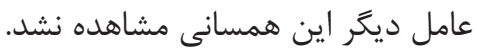

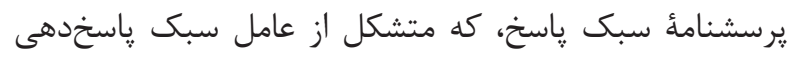

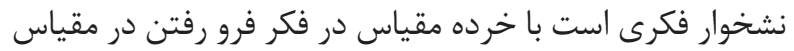

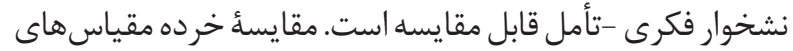

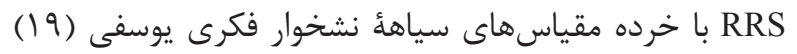

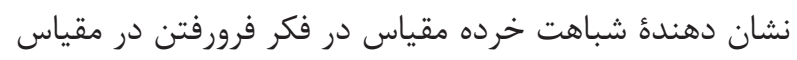

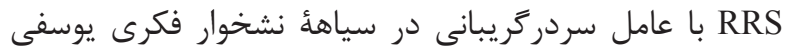

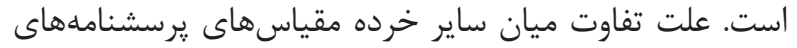

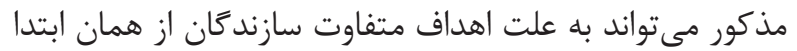

باشد كه باعث تفاوت در حيطه بندى سؤالات مى مئل أنود. مقايسُٔ خرده مقياسهاى يرسشنامهُ نشخوار فكرى-تأمل با خرده

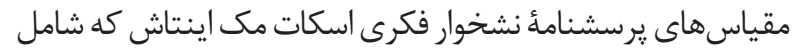

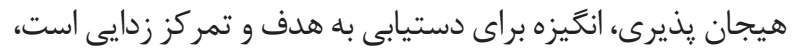

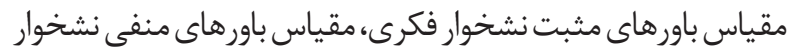

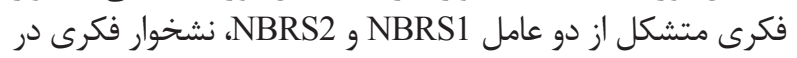

بحث و نتيجه كيرى

هدف يزوهش حاضر بررسى شاخصهاى روانسنجى مقياس

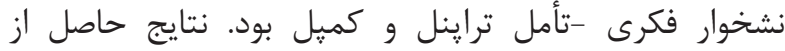

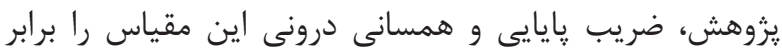

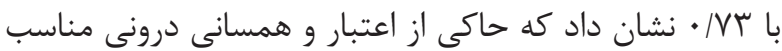
اين مقياس است.

نتيجهُ به دست آمده از اين يزوهش باضريب اعتبار يرسشنامههايى

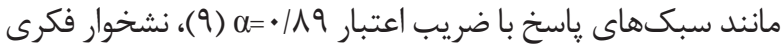

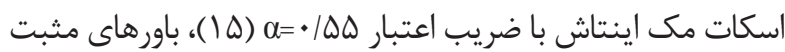

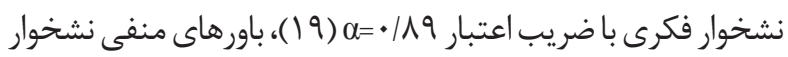

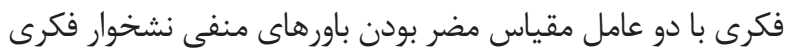

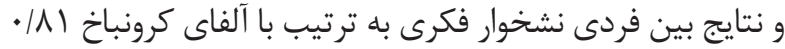

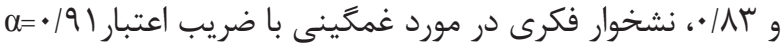

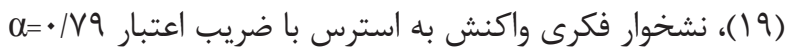

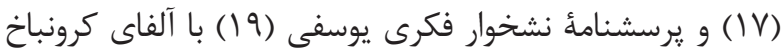

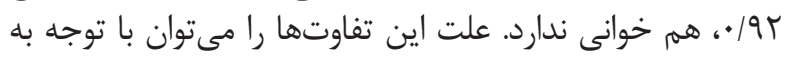

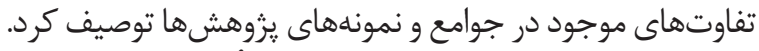

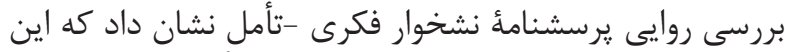

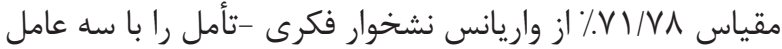
تأمل، درون نخرى و در فكر فرورفتن تبيين مى كند. بنابراين 
براساس نظريأ بِيشرفت هدف ساخته شده است كه به طور

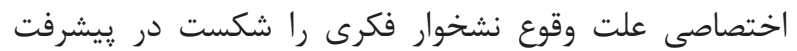

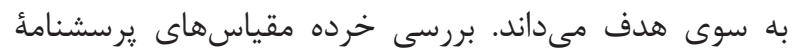

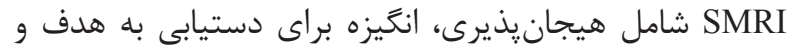

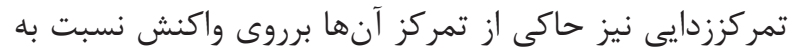

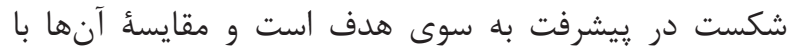

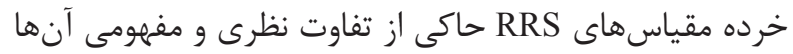

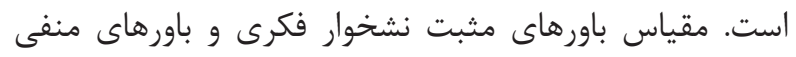

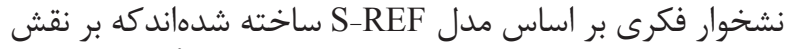

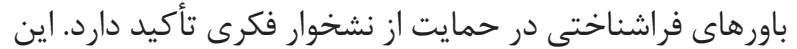

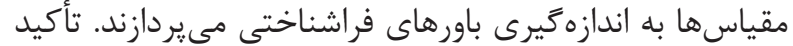

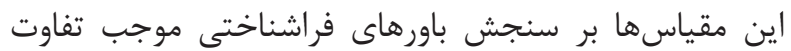

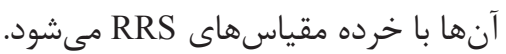

نشخوار فكرى واكنش به غمخينى تنها به اندازهذيرى نشخوار

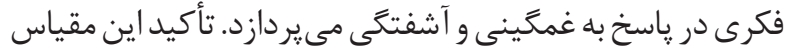

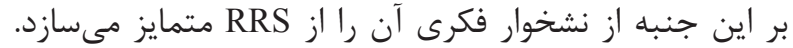

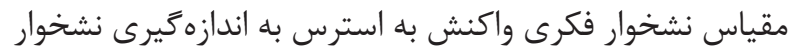

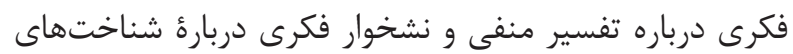

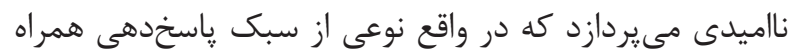

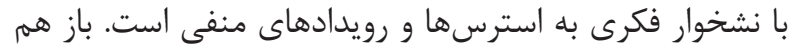

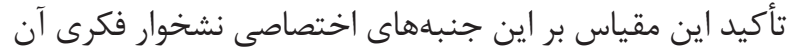
را از خرده مقياس هاى RRS متمايز مي ائسازد.

در اين يزوهش شاخصهاى روانسنجى مقياس نشخوار فكرى

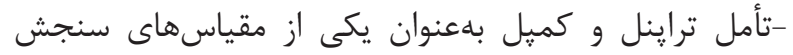

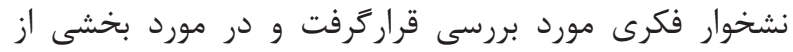

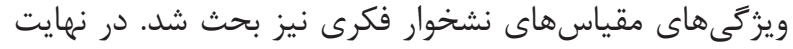

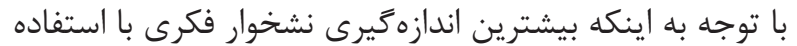

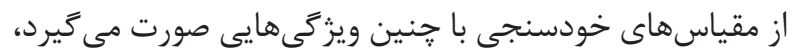

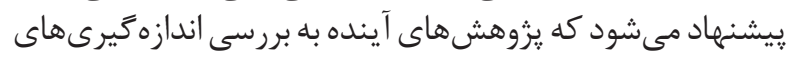

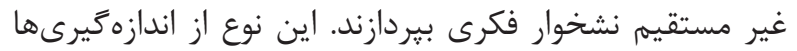

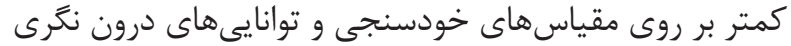

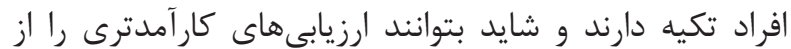

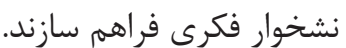

1. Ghorbani N, Watson PJ, Hargis MB. Integrative self-knowledge: correlations and incremental validity of across-cultural measure developed in Iran and the United States. J Psychol. 2008; 142(4): 395-412.

2. Rippere $\mathrm{V}$. What is the thing to do when you're feeling depressed? --a pilot study. Behav Res Ther. 1977; 15(2): $185-91$.

3. Robinson MS, Alloy LB. Negative cognitive styles and stress-reactive ruminationinte ract to predict depression: a prospective study. Cognit Ther Res. 2003; 27(3): 275-92.
مورد غمخينى كه سازماى تك عاملى است، يرسشنامهُ نشخوار فكرى

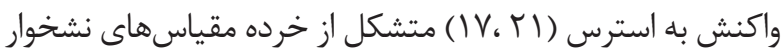

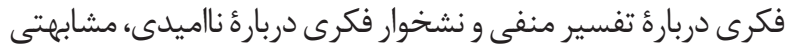

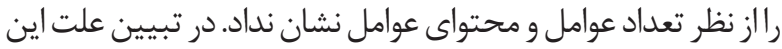

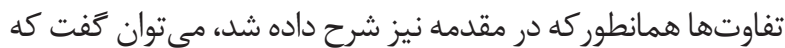

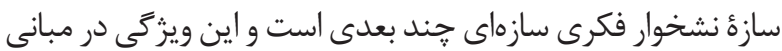

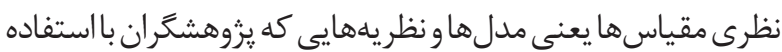

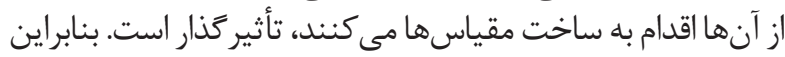

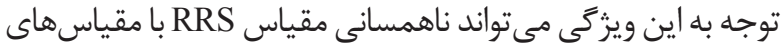

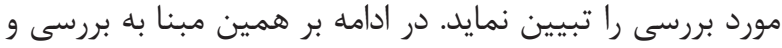

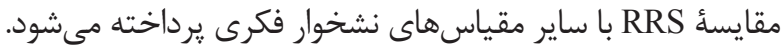

هدف تراينل و كميل از ساخت مقياس RRS تمايز ميان

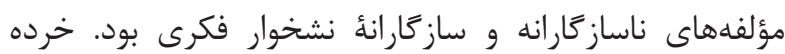

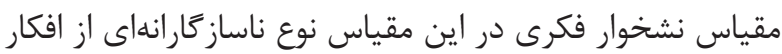

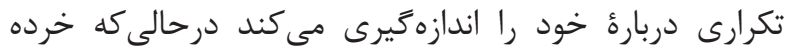

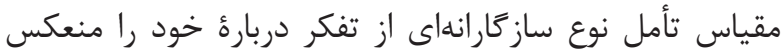

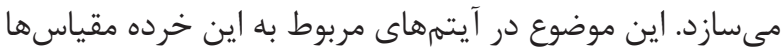

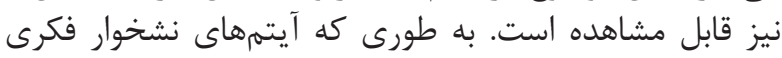

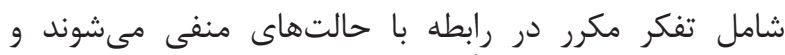

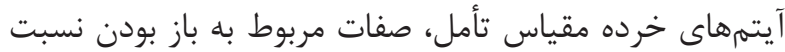

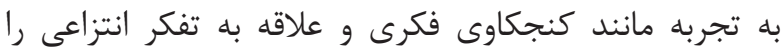

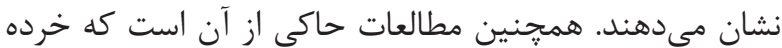

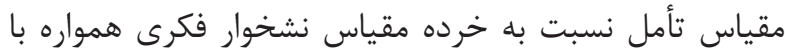

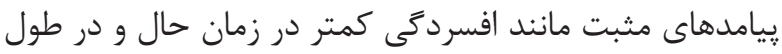

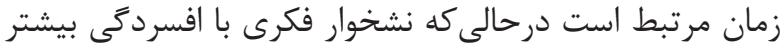

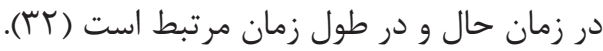

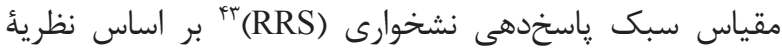

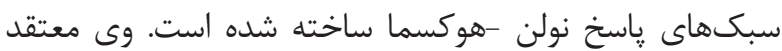

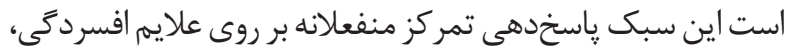

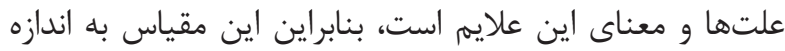

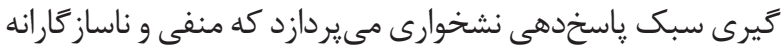

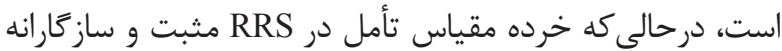

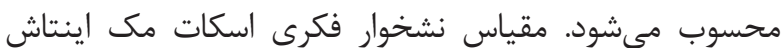

منابع

4. Martin LL, Tesser A. Some ruminative thoughts. Wyer RS. Advances in Social Cognition. $1^{\text {st }}$ ed. Lawrence Erlbaum Associates. 1996; P. 1-47.

5. Joormann, J. Differentioal effect of rumination and dysphoria on the inhibition of irrelevant emotional material: evidance from a negative priming task. Cognit Ther Res. 2006; 30: 149- 60.

6. Siegle GJ. Convergence and divergence in measures of rumination. Paper presented at the $34^{\text {th }}$ Annual

\footnotetext{
${ }^{43}$ Ruminative response style
} 


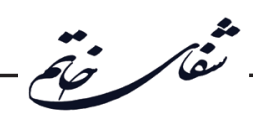

Convention of the Association for Advancement of Behavior Therapy. New Orleans. 2000.

7. Nolen-Hoeksema S. Responses to depression and their effects on the duration of depressive episodes. J Abnorm Psychol. 1991; 100(4): 569-82.

8. Garnefski N, Kraaij V, Spinhoven P. Negative life events, cognitive emotion regulation andemotional problems. Pers Individ Dif. 2001; 30(8): 1311-27.

9. Nolen-Hoeksema S. Morrow J. A prospective study of depression and posttraumatic stress symptoms after a natural disaster: the 1989 loma prieta earthquake. J Pers Soc Psychol. 1991; 61(1): 115-21. 10. Just N, Alloy LB. The response style theory of depression: tests and an extension of the theory. J Abnorm Psychol. 1997; 106(2): 221-9.

11. Spasojevic J, Alloy LB. Rumination as a common mechanism relating depressive risk factors to depression. Emotion. 2001; 1(1): 25-37.

12. Donald TZ. Rmination about stressful life event: measuring post-event rumination. MS Thesis. Washington State University, Department of Psychology. 2008.

13. Smith JM, Alloy LB. A roadmap to rumination: a review of the definition, assessment, and conceptualization of this multifaceted construct. Clin Psychol Rev. 2009; 29(2): 116-28.

14. Scott VB, McIntosh WD. Development of a trait measure of ruminative thought. Pers Individ Dif. 1999; 26(6): 1045-56.

15. Siegle GJ, Moore PM, Thase ME. Rumination: one construct, many features in healthy individuals, depressed individuals, and individuals with lupus. Cognit Ther Res. 2004; 28(5): 645-68.

16. Wells A, Matthews G. Modeling cognition in emotional disorders: the S-REF model. Behav Res Ther. 1996; 34(11-12): 881-8.

17. Wells A. Matthews G. Attention and emotion: a clinical perspective. $1^{\text {st }}$ ed. Psychology Press. 1995.

18. Papageorgiou C, Wells A. Depressive rumination nature, teory and tretment. $1^{\text {st }}$ ed. Wiley. 2004.

19. Yousefi Z, Abedi MR, Bahrami F, Mehrabi A. Construction and standardization of depressive rumination. Psychology. 2009; 13 (1): 54-68.

20. Conway M, Csank PA, Holm SL, Blake CK. On assessing individual differencesi $\mathrm{n}$ rumination on sadness. J Pers Assess. 2000; 75(3): 404-25.

21. Alloy LB, Abramson LY, Hogan ME, Whitehouse WG, Rose DT, Robinson MS, et al. The templewisconsin cognitive vulnerability to depression project: lifetime history of axis i psychopathologyin individuals at high and low cognitive risk for depression. J Abnorm Psychol. 2000; 109(3): 403-18.

22. Abramson LY, Metalsky GI, Alloy LB. Hopelessness depression: a theory-based subtype of depression. Psychol Rev. 1989; 96(2): 358-72.

23. Yousefi Z. Effectiveness of attention training in reducing depressive rumination of femalepatients. MS Thesis. Isfahan. University of Isfahan, Faculty of Education and Psychology. 2004.

24. Whitmer AJ, Banich MT. Inhibition versus switching deficits in different forms of rumination. Psychol. Sci. 2007; 18: 546-53.

25. Lyubomirsky S, Nolen-Hoeksema S. Effects of self-focused rumination on negative thinking and interpersonal problem solving. J Pers Soc Psychol. 1995; 69(1): 176-90.

26. Nolen-Hoeksema S. The role of rumination in depressive disorders and mixed anxiety/depressive symptoms. J Abnorm Psychol. 2000; 109(3): 504-11.

27. Rusting CL, Nolen-Hoeksema S. Regulating responses to anger: effects of rumination and distraction on angry mood. J Pers Soc Psychol. 1998; 74(3): 790-803.

28. Cheung MSP, Gilbert P, Irons C. An exploration of shame, social rank and rumination in relation to depression. Pers Individ Dif. 2004; 36(5): 1143-53.

29. Nolen-Hoeksema S, Jackson B. Mediators of the gender difference in rumination. Psychol Women Q. 2001; 25(1): 37-47.

30. Madahi MI. Rational behavior therapy-emotional rumination on depression and anxious people. Thought and Practice. 2012; 25: 43-56.

31. Trapnell PD, Campbell JD. Private selfconsciousness and the fivefactor model ofpersonality: distinguishing rumination from reflection. J Pers Soc Psychol. 1999; 76(2): 284-304.

32. Treynor W, Gonzalez R, Nolen-Hoeksema S. Rumination reconsidered: a psychometric analysis. Cognit Ther Res. 2003; 27(3): 247-59. 